\title{
Simbolismo y presencia subversiva del agua en L'Ombre de Venceslao y La Tour de la Défense de Copi
}

Vásquez-Sáenz, Henry F.

Universitat de València, Henry.Vasquez@uv.es

\begin{abstract}
Resumen
Raúl Damonte Botana, conocido artísticamente como Copi, fue un escritor, dramaturgo y dibujante argentino (1939-1987) que se instala definitivamente en París en 1962 (donde había vivido de niño con su familia exiliada). Allí escribió y publicó en francés la mayor parte de sus obras hasta su muerte. Dentro de su gran variedad literaria (teatro, novela, cómic...) resaltamos dos obras de teatro en las que el agua es un motivo recurrente que condiciona la intriga y los espacios donde evolucionan los personajes. En L'Ombre de Venceslao (escrita en 1977 y representada en 1978 en el Festival de La Rochelle), el agua aparece en espacios abiertos y está en permanente contacto con los personajes. Se trata de un elemento omnipresente que, a pesar de generar vida, se transforma en un componente devastador o destructor que incita a la muerte. En La Tour de la Défense (publicada en 1974 y representada en el Théâtre Fontaine de París en 1981), nos encontramos en un espacio cerrado e íntimo donde el agua es un elemento ambiguo que apasiona e inquieta; por un lado, despierta los deseos sexuales más desenfrenados $y$, por otro, propicia la aparición de bestias escalofriantes destinadas a ser devoradas.
\end{abstract}

Palabras clave: Copi; teatro; agua; simbolismo; subversión.

\section{Résumé}

Raúl Damonte Botana, connu artistiquement comme Copi, a été un écrivain, dramaturge et dessinateur argentin (1939-1987) qui s'est installé définitivement à Paris en 1962 (où il avait déjà vécu pendant son enfance avec sa famille en exil). C'est là qu'il a écrit et publié en français la plupart de ses œuvres jusqu'à sa mort. De sa production riche et variée (théâtre, roman, bande dessinée), nous voudrions mettre en valeur deux pièces de théâtre où l'eau constitue un motif récurrent qui conditionne l'intrigue et les espaces où évoluent les personnages. Dans L'Ombre de Venceslao (écrite en 1977 et créée en 1978 au Festival de La Rochelle), l'eau apparaît dans des espaces ouverts et elle est en contact permanent avec les personnages. En effet, il s'agit d'un élément omniprésent qui, même s'il est générateur de vie, devient un composant dévastateur ou destructeur incitant à la mort. Dans La Tour de la Défense (publiée en 1974 et créée au Théâtre Fontaine à Paris en 1981), nous nous trouvons dans un espace fermé et intime où l'eau est un élément ambigu, passionnant et inquiétant en même temps. D'une part, elle éveille les désirs sexuels les plus effrénés et, d'autre part, elle favorise l'apparition de bêtes terrifiantes destinées à être dévorées.

Mots-clés: Copi; théâtre; eau; symbolisme; subversion.

\footnotetext{
Abstract

Raúl Damonte Botana known artistically as Copi was an Argentinian author, playwright and cartoonist (1939-1987) who set up his permanent home in Paris in 1962 where previously with his exiled family he had lived as a child. He wrote and published his works there mainly in French until his death. His artistic production was varied, and amongst the plays, novels and comics two theatre productions stand out where water plays a recurring theme that affects both the intrigue and the spaces in which the characters develop. In L'Ombre de Venceslao (written in 1977 and performed in La Rochelle Festival in 1978) water appears in wide open spaces and the characters are permanently in contact with it. Water is seen as a boundless element which although generates life can also play a destructive part that leads to death.
} 
In La Tour de la Défense (published in 1974 and represented in the Théâtre Fontaine in Paris in 1981), we find ourselves in an enclosed and intimate space where water is an ambiguous element firing up passion and fear, on the one hand it awakens the most uncontrollable sexual desires and on the other it gives rise to the appearance of bloodcurdling beasts destined to be devoured.

Keywords: Copi; theatre; water; symbolism; subversion.

\section{Introducción}

El agua y su simbología es un aspecto poco abordado en el teatro de Copi. Este trabajo tiene como objetivo principal intentar desvelar, mediante un análisis interpretativo, la pertinencia de este elemento a través de dos de sus obras: L'Ombre de Venceslao y La Tour de la Défense. Entendiendo el agua como un fluido en movimiento y en permanente cambio en el imaginario del autor, haremos, en primer lugar, una descripción sintética de los espacios para explicar la elección de estas dos obras que aparecen unidas por el agua y separadas por el lugar geográfico y la temática. Seguidamente intentaremos dilucidar cómo el agua interfiere directamente en el comportamiento de los personajes apropiándose no solo del espacio físico en el que se mueven sino también de sus espacios psicológicos. Y finalmente veremos de qué manera el agua, que cambia repentinamente de estado transformándose en grandes tormentas que provocan múltiples desastres, se convierte a su vez en un elemento purificador o regenerador. En definitiva, el elemento acuático en sus múltiples facetas forma parte de un ritual altamente corporal donde los binomios agua/vida y agua/muerte simbolizan el recorrido ineluctable de los personajes hacia un trágico destino.

\section{1. "Cuando el río suena, agua lleva»}

Muchos son los filósofos, científicos y escritores que han indagado en el significado profundo del agua como parte de una determinada lógica cósmica y fruto igualmente de las más variopintas fantasías. Copi no podía escapar a estos dos aspectos complementarios que aúnan realidad natural y desbordada imaginación.

En este sentido L'Ombre de Venceslao y La Tour de la Défense serían, si se nos permite el uso metafórico, dos obras hídricas. Los espacios que en ellas se escenifican están separados cultural, temporal e hídricamente por un océano: el Atlántico y dos continentes: América (del Sur) y Europa. La primera, dividida en dos actos, con dieciséis escenas cada uno, nos sitúa en la provincia de Entre Ríos en Argentina, además de Buenos Aires y las cataratas del Iguazú. Es una historia de amor, desgracia y muerte que pone en escena a una familia compuesta por Venceslao y su hija China, por un lado, y de su amante Mechita y su hijo Rogelio (cuyo padre es supuestamente Venceslao), por otro. También aparece la figura de su vecino, el viejo Largui, enamorado de Mechita igualmente. Todos viven en Diamante, un pueblo humilde de Entre Ríos a principios del siglo XX.

VENCESLAO.- Ecoute, Perroquet ! Toi qui est un animal de grande résistance et qui se souvient du parler de la Bande Orientale, enregistre bien ce que je vais te dire, il se peut qu'un jour quelqu'un l'entende et puisse se faire une idée de ce que fut ce temps-là. Tu te souviendras que je suis natif de la ville de Trente-Trois, en Uruguay, fils d'un Juarez et d'une indigène tabaré, appelée Abayubá. C’est dans ma maison que la milice du coin l’a égorgée, aux environs de 1850. Et moi, comme j'étais le plus petit de tous, j'ai pu sauver ma vie en me cachant dans un puits, et alors une certaine Mireya me prit en affection, elle me recueillit et m’apprit à baiser, tout gamin que j'étais. Avant le début du siècle, la solitude, c'était pas une invention. On vivait avec elle, les gens, ils existaient, on se laissait pas aller comme aujourd'hui, et moi, je suis de ce temps-là. Quand j'ai été jeune homme, la Mireya m’offrit un poulain blanc et j'ai traversé le fleuve Uruguay à la nage, accroché à sa crinière. Et on a pris le large au grand galop jusqu'à Diamante, le grand bourg de l'époque ; là, on s'est fait engager dans l'hacienda du vieux Jacinto Parra, et il m'offrit sa fille. La suite, tu la connais, Roquet (Copi, 1999a: 48-49).

Sometidos a las inclemencias y dificultades del medio natural y social en el que habitan, la peculiar familia está predestinada a tomar dos rumbos diferentes que dan lugar a dos historias paralelas: la que inician los que emprenden un recorrido por «les chemins de l'exode» (Copi, 1999a: 25) hacia las cataratas del Iguazú y la que llevan a cabo los que 
parten hacia sociedades más «desarrolladas», en este caso Paraná, la capital de la provincia, y luego la gran capital, Buenos Aires. En esta obra aparecen temas recurrentes en Copi como la libertad, la identidad y el machismo, además de los conflictos políticos, religiosos y la desigualdad social. La segunda obra, dividida en dos actos, sin escenas, nos sitúa en París, una ciudad emblemática bañada externamente por el río Sena e internamente por todo un circuito de tuberías, conductos y alcantarillados. El dramaturgo nos sumerge en una inquietante intriga protagonizada por una pareja homosexual, Jean y Luc, una joven madre asesina, Daphnée, que está siempre «sous acide», un travesti mitómano, Micheline, y un joven árabe seductor, Ahmed. Todos se dan cita en casa de Jean y Luc, en el piso trece de un lujoso apartamento de una de las torres del barrio de la Défense para compartir la cena de nochevieja en la transición de 1976 a 1977.

LUC.- C’est le premier de l'an.

DAPHNÉE.- Oh, c’est vrai, soixante-dix-sept! On entend les pétards !

LUC.- Oui, c'est la fête, enfin, la fin de la fête.

DAPHNÉE.- Tu es intelligent, toi. C’est peut-être ce qui m’a séduit le plus chez toi. Ça et l’odeur de tes cheveux. Et toute ton odeur, ça m'a rendue folle depuis que je te connais, quoi. L'odeur de tes aisselles, de tes pieds. C'est pour ça que je passais mon temps fourrée ici. Je volais tes vêtements sales, tu vois, et je m'endormais avec le nez fourré dedans. Ça m’a absorbée complètement, je suis devenue folle... (Copi, 1999b: 171-172).

En esta intriga prevalece igualmente el problema de la soledad, la identidad, la igualdad e integración de comunidades minoritarias, imbricándose con aspectos políticos y religiosos, además de un ataque directo al mercado del consumo y a las nuevas sociedades capitalistas.

Esta separación cultural entre ambas obras también conlleva una diferencia en el tratamiento de los espacios donde se desarrollan las acciones. En Argentina asistimos generalmente a espacios abiertos y exteriores (salvo algunas excepciones), lo que permite al autor hacer hincapié en la fuerza natural, devastadora y destructora del agua. En este sentido el director Jorge Lavelli (2015, on line) en una entrevista de Boris Horvat afirma que está trabajando actualmente con el Centre Français de Promotion Lyrique sobre una ópera que se estrenará en 2016/2017 basada en esta pieza de Copi de la que precisa que «c'est un voyage avec une partie des personnages qui vivent à Parana au milieu des inondations et des tempêtes de la pampa». El agua, en su estado natural con un poder potencialmente devastador, es un elemento que vemos de principio a fin en sus múltiples facetas y manifestaciones: nubes, gotas, llovizna, lluvia, chaparrones, tormentas, arco iris, vendavales, riachuelos, ríos, arroyos, inundaciones... En cambio, en una de las torres de la Défense, en París, asistimos a un espacio cerrado y asfixiante donde el agua aparece continuamente, no en su vertiente de elemento natural de contundente y evidente fuerza destructora a la que hemos aludido, sino como un sutil fluido igualmente inquietante pero dócil y de fácil manipulación. Esta característica del agua encauzada o supuestamente controlada, condiciona tanto ese espacio cerrado como la propia intriga, convirtiéndose en uno de los elementos principales de la acción. En este sentido destacaríamos en primer lugar el aspecto «agua-seducción», en tanto que elemento hipnotizador que atrae a los personajes para dar rienda suelta a sus deseos sexuales; en segundo lugar el «agua-caos», ya que facilita que los personajes entren en un estado de histeria, delirio y locura colectiva que crea a su vez ese efecto de confusión tan característico del autor; y por último, el «agua-autodestrucción», puesto que los personajes pierden el control de sus acciones haciendo que todo lo que gira en torno al agua se transforme en aniquilación, ya sea la muerte casual o la intencionada. Así pues, pasamos de las grandes catástrofes naturales del río Paraná: «Putain de merde, l’inondation ! Putain de merde, l’inondation !» (Copi, 1999a: 18) al pequeño cuarto de baño del apartamento de la Défense, un lugar misterioso y privilegiado donde todo puede ocurrir: diferentes escenas amorosas, de alta carga erótica, la aparición de bestias escalofriantes, o cuanto menos sorprendentes (una serpiente que ha engullido una rata, la llegada de una gaviota perdida en la oscuridad) y hasta el lavado ritual del cadáver de Katia, la hija de Daphnée. De este modo el agua pierde su supuesta pureza para convertirse en un fluido contaminado que se mezcla con la sangre tiñéndolo todo de rojo e incitando al deseo: «Tout est inondé de sang [...] La baignoire est pleine de sang» (Copi, 1999b: 91). En este sentido Ezequiel Lozano precisa que «la limpieza de la sangre bajo el agua transforma la sangre y la higiene en sexo desenfrenado» (Lozano, 2012: 61). 
No obstante, entre catástrofes naturales por un lado, violencia y caos de lo cotidiano por otro, la genialidad del autor está en la conjugación acción-lenguaje para hacer que lo extraordinario y trágico se convierta en habitual e intrigante. El ritmo frenético y la rapidez con la que las acciones se entrelazan y se suceden da la impresión de asfixia y descontrol pero, al mismo tiempo, el tono contrastado hace que pierdan intensidad. De este modo la palabra, que vehicula un humor negro, en ocasiones altamente corrosivo, se impone gracias a los discursos directos y espontáneos que restan importancia a la gravedad de los acontecimientos creando en el lector-espectador una sensación ambivalente de sorpresa y risa pero a la vez de impacto y desconcierto. En este sentido Jorge Luis Montiel (2013, on line), con motivo de la representación en español en el Teatro Nacional Cervantes de Buenos Aires en 2013 de La sombra de Wenceslao, dirigida por el conocido actor, director de teatro y escritor uruguayo Villanueva Cosse, afirma: «En ella Copi parodia personajes autóctonos con un humor ácido y un lenguaje explícito que puede llegar a incomodar a un espectador desprevenido». Escuchemos, a título de ejemplo, la voz de Rogelio, encerrado en un baño y a punto de morir envenenado: «Putain, quelle chiasse j’ai attrapée ! Et qu’est-ce que je peux péter ! [...] Aïe, putain de douleur ! La chiasse me reprend ! Mon Dieu, je chie du sang ! Au secours ! Ils m’ont empoisonné ! [...] Ouvrez, je vous en prie, je vous en prie, je suis en train de crever [...] !» (Copi, 1999a: 53). Precisamente sobre este uso «obsceno» del lenguaje en el teatro de Copi, Lionel Souquet señala:

C’est justement le caractère incroyablement ordurier du langage (par un cumul de la vulgarité et de l'obscénité, dans le signifié et le signifiant, qui heurte la bienséance du public bien-pensant) qui le fait jaillir sur scène [...] comme une langue étrange, presque étrangère, si argotique qu'elle se détache de la banalité quotidienne, du langage ordinaire, et en devient littéraire, voire lyrique mais tout en restant dans l’oralité, gardant une certaine crédibilité comme langue « vivante », comme langue de vie (Souquet, 2013: 135).

Este estilo particularmente subversivo y transgresor que caracteriza a Copi aparece igualmente en La Tour de la Défense cuando vemos, por ejemplo, que en la cena de Nochevieja el cordero, que tiene claras connotaciones religiosas y que se quema en el horno, se sustituye por una serpiente que aparece en el baño (igualmente cargada de simbolismo religioso). No es tampoco inocente que sea el personaje de Ahmed quien incite a comer la serpiente (que lleva una rata en su vientre), cocinada al horno igualmente: «Goûtez ça, goûtez ça, ça c’est l’anus du serpent. Tu vois comment il est rond ? Les serpents n'ont qu'un seul trou, à part la bouche. C’est par là que ça baise et que ça pond les œufs. Regarde comme c’est élastique un cul de serpent ! Tiens Luc, C’est pour toi !» (Copi, 1999b: 111).

Así observamos cómo Copi en estas dos obras nos muestra, por un lado y desde las peripecias de una humilde familia de gauchos en L'Ombre de Venceslao, la difícil pero gratificante adaptación e interacción entre el hombre y la naturaleza: «L'avantage de vivre à côté de ces chutes c'est qu'on voit toujours l'arc-en-ciel qui est si joli» (Copi, 1999a: 38); incluso si su destino final es la muerte, como en el caso de Venceslao que da fin a su existencia terrenal al lado de las cataratas del Iguazú: «J'en suis pas sûr, Mechita, mais j’ai comme l’impression que je vais mourir. Tu me mettras une croix en chêne, hein, tout près des chutes ?» (Copi, 1999a: 45). Y por el otro, en La Tour de la Défense, se invierten los papeles de dominio. En París, el agua no tiene la misma fuerza sobre el hombre ya que este elemento está domesticado y adaptado a las necesidades básicas para la subsistencia. El agua requiere un tratamiento y participa en un complejo mecanismo de tuberías y procesos de purificación para su consumo y manipulación, sobre todo en el cuarto de baño. También vuelve de manera cíclica a los desagües y cañerías que la conducen al alcantarillado, los famosos égouts parisiens, espacios cerrados y cavernosos en los que el agua se convierte en un elemento contaminado y de refugio de todo tipo de criaturas terroríficas como ratas y serpientes: «Ça se promène partout dans les grands immeubles. Ça cherche la chaleur dans les tuyaux des chauffages, alors ça sort parfois par l'égout des baignoires, parce que dans les grands immeubles toute la tuyauterie communique» (Copi, 1999b: 88). Así, en esta obra, Copi juega con la imaginación del lector-espectador y hace que salgan a la superficie toda una serie de «monstruos» que viven en constante movimiento bajo el espacio de los humanos, donde impera el convencionalismo. Las tuberías y el agua limpia/sucia establecen misteriosas conexiones entre dos mundos y dos realidades sociales que oponen lo que se muestra y lo que se oculta. De esta forma, el Sena, lejos de representar un río poético e idílico, se convierte en un vertedero de bestias e inmundicias, reales y simbólicas, de una sociedad caótica y podrida. 


\section{2. «Del agua mansa líbreme Dios, que de la brava me libraré yo»}

Como hemos señalado, el agua es un elemento que interfiere continuamente en las acciones de los personajes ya sea en espacios exteriores o interiores. En L'Ombre de Venceslao observamos que, desde un vulnerable pueblo, Copi da una visión cosmogónica del paisaje argentino donde el agua aparece como principio de vida pero también de destrucción, idea bíblica que nos recuerda al gran diluvio universal. Incluso podemos interpretarla desde la concepción del agua como elemento fecundador de la tierra a través de la lluvia, una simbología no exenta de connotaciones eróticas. En este sentido Copi seguiría a su manera una tradición muy arraigada en la literatura hispanoamericana. Baste recordar al gran poeta chileno Pablo Neruda y su Canto General (1950) donde la naturaleza y el agua del continente americano están muy presentes. En Copi, esta presencia del agua salvaje y descontrolada representaría a la naturaleza de América, amenazante y temible, presagio de terribles acontecimientos: «V'là l'inondation ! V'là l'inondation !» (Copi, 1999a: 26); «[...] quelle horreur ! [...] Je suis nerveux ! Je suis nerveux !” (Copi, 1999a: 27); "Encore le tonnerre! Je suis terrorisée. Y a plus qu’à prier. [...] Tonnerre, éclairs! Un désastre terrifiant!» (Copi, 1999a: 31), «On se noie ! [...] Au secours! Le courant m’emporte ! [...] Ô mon Dieu !» (Copi, 1999a: 32). Por el contrario, en La Tour de la Défense, pasamos de la aldea argentina rodeada de exuberante naturaleza a la gran ciudad europea que es París y más concretamente al espacio cerrado y claustrofóbico, a pesar del lujo, de un apartamento en el que están atrapados los personajes: «Je peux sortir la tête par la fenêtre ?» (Copi, 1999b: 113). Las ventanas son una mera ilusión de escapatoria ya que los que intentan abandonar dicho espacio o vuelven heridos (es el caso de Luc) o mueren estrellándose contra otro edificio incendiado (todos excepto Micheline y Ahmed que observan por la ventana):

Une explosion dehors. Ils courent à la fenêtre.

MICHELINE.- Oh, Ahmed, regarde, regarde, la voiture se dirige vers la tour à toute vitesse !

AHMED.- Elle rentre dedans, putain de merde ! Ils brûlent ! Ils brûlent ! Ils sont tous morts ! Ils sont tous morts !

(Copi, 1999b: 182).

En esta obra, el agua está presente sobre todo en el microespacio del baño, a través de las duchas en la bañera básicamente, pero también se hace alusión a ella en su estado sólido, debido a la congelación: «Ça se défait, ça devient de l'eau [...] C'est des cristaux d'eau! Tiens, regarde, c'est dans le Larousse: neige. La neige vue au microscope. C'est ça ce qui se dissout à la chaleur de ta main, des cristaux microscopiques [...]» (Copi, 1999b: 112-113). El agua está caracterizada por su movimiento pero se establece una oposición entre el agua encerrada que corre por las tuberías y desagües del apartamento y el agua exterior que cae del cielo en forma de nieve. La primera corresponde con el ritmo acelerado de la acción que genera desorden, caos y confusión en contraste con la suave caída de la segunda en el exterior cuya contemplación calma este ritmo frenético propiciando por un breve instante la ensoñación, la reflexión y el afloramiento de los recuerdos. Se produce pues un contraste entre agua prisionera (interior) y agua libre (exterior), entre enclaustramiento y libertad. En cualquier caso, el fuego como elemento devorador (y en cierto sentido regenerador) terminará triunfando de alguna manera sobre el agua al provocar el desvanecimiento de la nieve cuando entra en contacto con las llamas de las torres que se están incendiando o con el contacto corporal.

Por otra parte, querríamos destacar que a lo largo de la obra asistimos, como marcan las acotaciones, a un continuo trasiego de idas y venidas al cuarto de baño por diferentes motivos, lo que hace que la aparición del agua intensifique las acciones de los personajes en un espacio que, en principio, está concebido para ser un lugar íntimo, de relajación y aseo personal. Vemos como, una vez en el baño, el espacio de la acción se va reduciendo y concentrando en el baño, convertido en el epicentro de diferentes sucesos como lavar un dedo cortado, en este caso el de Jean: «Aïe, merde, je me suis coupé» (Copi, 1999b: 72), o limpiar una herida en la frente de Luc: «Je n’ai pas tenté de me suicider. J’ai ouvert la porte de l'ascenseur par erreur entre deux étages» (Copi, 1999b: 73). El espacio del cuarto de baño, con su bañera en un lugar preponderante, adquiere una gran carga erótica y sirve también de inspiración para los encuentros eróticos entre Jean y Luc o Micheline y Ahmed: 
AHMED.- Micheline, une serviette !

MICHELINE.- J'arrive mon grand ! (À Jean:) Tu as vu comme c'est pas un rêve ? C’est une réalité. (Elle va dans la salle de bains.) Tiens, mon chou, il n'y a pas de serviette, viens que je te sèche les cheveux avec mon mouchoir! Mais arrête, qu'est-ce que tu fais, tu es fou?

VOIX D’AHMED.- Viens dans la baignoire !

VOIX DE MICHELINE.- Attends qu’on soit chez moi, mon chéri, je vais pas me faire violer dans une baignoire !

On entend des fous rires, des phrases dans le genre: non, mais pour qui te me prends; des bruits d'eau.

JEAN.- Salope ! (Il va fermer la porte de la salle de bains. Il va a la fenêtre) (Copi, 1999b: 160).

Pero el baño se convierte de repente en un espacio escalofriante con la aparición de la boa constrictor que alberga una rata en su interior, según César Aira los «animales favoritos» de Copi (Aira, 1991: 121): «Aââââ, un serpent !» (Copi, 1999b: 88). En el baño lavarán igualmente una gaviota para intentar revivirla y en la bañera limpiarán el cadáver de Katia, la hija de la vecina y amiga Daphnée, asesinada por ésta y encerrada en una maleta de viaje.

Más allá de este análisis del agua en sus diferentes estados y espacios, que influyen de manera directa en los personajes, encontramos otras posibles interpretaciones de la presencia del agua más abstractas y complejas. Frente al simbolismo del agua en movimiento a través de campos abiertos, se opone el agua estancada en espacios cerrados. En L'Ombre de Venceslao, los recorridos naturales y las bifurcaciones dibujadas por el agua son también una metáfora de los éxodos humanos que hacen referencia, a su vez, a acontecimientos históricos y militares que se remontan a la época de la emancipación argentina y del Virreinato del Río de la Plata. Estas referencias históricas se ejemplifican claramente en la disgregación y dispersión de la familia de Venceslao, prototipo de gaucho errante decimonónico con ansias de libertad, figura que Copi recupera y subvierte a su manera, con grandes dosis de humor y sexualidad: «c'est dans la solitude de la pampa et sa monotonie sauvage que se construit le caractère introspectif et sombre de son légendaire habitant. Il parcourt l'étendue infinie de la plaine et il ne fait qu'un avec son cheval» (Lavelli, 1999: 7). Se trata de un desplazamiento simbólico y cíclico, que sigue los pasos del viejo Artigas, referencia clara al general José Gervasio Artigas (1764-1850) figura clave de la independencia y el federalismo a uno y otro lado del Río de la Plata.

CHINA.- Comment ça, papa, et tu pars tout seul ?

VENCESLAO.- Pour l'instant, j’en sais rien. Donne-moi mon chapeau, China, et mon foulard.

ROGELIO.- Mais, comment vous partez vers le Nord, monsieur ? Et où ça ?

VENCESLAO.- Par les chemins de l'exode du vieil Artigas. Adieu, mes enfants, je vous donne ma bénédiction ! (Copi, 1999a: 25).

Así Venceslao y su amada Mechita emprenden este recorrido desde Diamante hasta las Cataratas del Iguazú, lugar idílico y mítico, separador de dos mundos como si de la laguna Estigia se tratara, donde decide deliberadamente dar paso a la muerte, un suicidio que intenta enaltecer y llevar a lo más sublime ya que, según él, hombre y naturaleza forman un todo: «Voilà, je mets ma chemise blanche du dimanche pour me pendre, comme ça, vous n'aurez pas à me changer une fois mort ! [...] Adieu, petit Roquet ! Et toi, ma terre, bonsoir ! Il se pend» (Copi, 1999a: 49). No obstante, como en los mejores dramas de teatro barroco, Venceslao resucita de entre los muertos y reaparece en forma de sombra. Se convierte en un espectro tranquilizador que dialoga más allá de la muerte con su amada Mechita:

MECHITA.- Mais où es-tu, Venceslao ?

VENCESLAO.- J'en sais rien; je sais seulement que je n’ai plus les pieds sur terre.

MECHITA.- Tu es tout seul ?

VENCESLAO.- Je sais pas. S’il y a quelqu'un, je ne peux pas le voir.

MECHITA.- Tu crois que quand je mourrai, je pourrai être avec toi, Venceslao ?

VENCESLAO.- Toi, tu seras toujours avec moi, la vieille (Copi, 1999a: 55). 
En cuanto a sus hijos, China y Rogelio, emprenderán un recorrido opuesto desde Diamante a Paraná y luego a Buenos Aires, sociedad «civilizada», que los hipnotiza, los ahoga y los engulle metafóricamente. Rogelio morirá envenenado por China, su hermanastra y amante -«j’aime Rogelio et je veux me marier avec lui» (Copi, 1999a: 19)-, quien, seducida por un director de cine, se deja drogar y convencer para cometer el crimen. Y esta, entre champán, drogas y conflictos políticos, muere en manos de aquella sociedad que ella misma buscaba con anhelo desde el inicio de la obra:

COCO.- [...] Gladys, passe-moi un sachet de cocaïne et la clé du boudoir.

CHINA.- Moi, je deviens complètement folle !

Des coups de feu.

VOIX DE GLADYS.- Tous à plat ventre ! Une fusillade !

VOIX DU CRIEUR DE JOURNAUX.- L’État de siège ! La Nación, La Nación, première édition ! L’État de siège est proclamé !

CHINA.- Mon Dieu ! Qu'est-ce qui se passe ? Ils m’ont tiré dessus !

Elle meurt dans l'escalier (Copi, 1999a: 54).

Respecto a La Tour de la Défense el agua no tiene libertad de movimiento al estar encerrada en tuberías y depende de la voluntad de los personajes que deciden en qué momento la liberan mediante los mecanismos de apertura de grifos, principalmente en la bañera. El agua, por su capacidad de estar parada y estancada, tiene un potencial de elemento contaminante y perturbador. Es precisamente este control sobre el elemento natural lo que contribuye a potenciar el caos y delirio de los personajes. Lo inesperado y lo extraño llega a través del agua y permanece como un elemento inquietante a lo largo de toda la obra. No es casual la aparición de la serpiente en la bañera, monstruo contemporáneo con evidentes resonancias mitológicas, empezando por el propio titán Océano, y con claras connotaciones sexuales: «La serpiente es un monstruo de las profundidades previo al nacimiento de los dioses» (Lozano, 2012: 10). Copi hace una vez más un uso subversivo de la serpiente en un medio acuático, convirtiéndola en una criatura de las alcantarillas que se pasea a sus anchas por cañerías y desagües urbanos, se alimenta de ratas y aparece inesperadamente en esa particular fiesta de nochevieja de 1976. La crítica social vinculada a una sociedad capitalista e individualista es evidente, el hecho de «desmitificar, desacralizar una imagen, es también una forma de desnudarse, un intento de quitar las máscaras que habitan en el inconsciente colectivo» (Rosenzvaig, 2003: 146). En este contexto y justo en el momento de la transición entre los dos años, la aparición de otro animal moribundo, próximo a las aguas marinas en libertad como es la gaviota, no es banal para la temática que nos ocupa y presagia un trágico final. En este sentido podemos afirmar junto a Ezequiel Lozano que Copi «bendice a sus monstruos y, a través de ellos, a todos los monstruos» (Lozano, 2012: 65).

\section{3. «Algo tendrá el agua cuando la bendicen»}

Entre la inesperada llegada de las devastadoras tempestades y la aparición de inquietantes criaturas relacionadas con el medio acuático, el autor también utiliza el agua como elemento purificador que forma parte de los rituales de limpieza de los cuerpos. Por un lado, Venceslao se entrega a todo un ritual de aseo íntimo antes de su partida hacia el Norte, precisamente el domingo, día del Señor, con las consabidas connotaciones religiosas:

VENCESLAO.- Apporte-moi la bassine du dimanche que je prenne un bain.

CHINA.- Papa, tu vas pas te mettre tout nu !

VENCESLAO.- T'as jamais vu les couilles d'un homme, hein ? Apporte-moi la bassine, China !

CHINA.- J'en ai marre de tes folies, papa ! 
VENCESLAO.- Et toi, file me chercher l'arrosoir, crétin ! Ne reste pas là, planté comme un con ! Toi, China, amènemoi le savon et la serviette, s'il te plaît.

CHINA.- Papa, je veux pas te voir tout nu !

VENCESLAO.- Frotte-moi le dos, China ! Et toi, Rogelio, va me chercher mon poncho de voyage dans l'armoire (Copi, 1999a: 24).

No obstante Copi, siguiendo su principio básico de ambigüedad y subversión de los significados, elige precisamente el domingo para el baño de Venceslao, cuestionando de este modo su carácter sagrado, ya que el personaje no parece creer más que en sí mismo y en el momento presente. Copi juega continuamente con la desacralización de lo sagrado: «Pas grâce à Dieu, China, non ! Grâce au Temps ! Hé ! temps ! Je te rends grâce !» (Copi, 1999a: 32). Ese baño tendría un claro carácter simbólico de transición y renacimiento, idea ligada al bautismo por lo demás y marca el camino del éxodo de Venceslao. Ese momento marcará también la transformación del personaje, la separación de la familia y el camino hacia la muerte, pero también hacia la verdad, la sabiduría y la libertad: «Crois-moi, je suis un homme pur» (Copi, 1999a: 22). En la Tour de la Défense el agua también se utiliza de manera ritual, principalmente para lavar y purificar el cadáver de la pequeña Katia:

LUC.- Bon, je vais laver Katia dans la baignoire et on va la coucher dans son berceau, elle aura l'air plus présentable pour John.

DAPHNÉE.- C’est à moi de le faire. [...] On me laissera l'enterrer moi-même ?

LUC.- Oui, Daphnée, c'est ta petite fille. Et à présent nous allons laver le petit corps avec de l'eau froide, ensuite on la frottera à l'eau de Cologne. Je vais t'aider.

DAPHNÉE.- Merci.

Ils entrent dans la salle de bains. On entend couler le robinet. Luc entre (Copi, 1999b: 148-149).

Copi, a través de lo que el propio personaje de Micheline califica de «spectacle sordide» (Copi, 1999b: 150) juega continuamente con los límites de lo representable y los convencionalismos para mostrar la vulnerabilidad humana, la depredación y la indiferencia social, todo inmerso en una serie de pequeños rituales de lo cotidiano donde el humor negro está muy presente.

\section{Conclusión}

En conclusión, hemos intentado demostrar en este trabajo que el agua juega un papel fundamental en estas dos obras muy diferentes de Copi, donde sin embargo encontramos elementos comunes que caracterizan al autor. En ambas el agua marca el ritmo de las acciones y también el destino de los personajes, estando presente en los momentos clave de cambio y metamorfosis. Un agua que está en permanente movimiento y transformación como la propia identidad fluida y transfronteriza de los personajes, ya sea en la salvaje pampa argentina en la transición entre el siglo XIX y el XX, ya sea en un elegante piso parisino en el paso de 1976 a 1977, ya sea en el propio Copi cuya vida transcurrió entre uno y otro lado del río de la Plata y entre una y otra orilla del océano Atlántico. Copi, el viajero, como lo calificaría Colette Godard, nunca está en un mismo sitio, está con nosotros y en su mundo: «Il est le héros de dangereux voyages. Il crée le cosmos silencieux qui le happe, l'océan qui le roule, l'ouragan qui l'entraîne dans le vertige de chutes sans fin et sans cesse répétées, jusqu'au fond de gouffres où il se retrouve face à lui-même, face à des monstres indestructibles» (Godard, 1999: 186) Y es que el teatro de Copi nos sitúa siempre en el «entre-dos» (aguas) y en la encrucijada (de caminos), un teatro donde nada es lo que parece.

VENCESLAO.- J'en suis pas sûr, j’en suis pas sûr. Faudra qu'on y réfléchisse, la vieille, parce que pour le moment, tout ça, je le vois si confus. Je commence à disparaître! Je commence à disparaître ! (Copi, 1999a: 55). 


\section{Referencias bibliográficas}

AIRA, César (1991). Copi, Rosario: Beatriz Viterbo.

Copi (1999a). L’Ombre de Venceslao. Montreuil: Théâtrales.

Copi (1999b). La Tour de la Défense. Paris: Christian Bourgois.

Godard, Colette (1999). «Copi le voyageur» en Copi. La Tour de la Défense. Paris: Christian Bourgois.

LAVELLI, Jorge (1999). «Préface» en Copi. L’Ombre de Venceslao. Montreuil: Théâtrales.

LAVELLI, Jorge (2015). Jorge Lavelli dans le Off, là où «on ne l'attend pas». < htttp://www.20minutes.fr/france/1688626-20150719avignon-jorge-lavelli-off-la-ou-on-attendait-pas> [Consulta: 08 de abril de 2016]

LozAno, Ezequiel (2013). «Cuatro abordajes de las sexualidades disidentes, una comparación» en III Congreso Internacional Cuestiones Críticas: 10. Rosario, Argentina.

LozAno, Ezequiel (2012). «Indagaciones queer a Copi a través de La Torre de la Defensa» en Apuntes de teatro N 136: 54-69. Santiago, Pontificia Universidad Católica de Chile.

MonTIEL, Jorge Luis (2013). La sombra de Wenceslao. <http://noticias.perfil.com/2013/09/13/la-sombra-de-wenceslao/> [Consulta: 03 de abril de 2016]

Neruda, Pablo (1990). Canto general, Madrid: Cátedra.

Rosenzvaig, Marcos (2003). Copi, Simulacro de espejos. Universidad de Málaga.

SouQuet, Lionel (2013). «Copi : l’Immoderato cantabile d’un Argentin francophone» en Morcillo, Françoise y Pélage, Catherine Littératures en mutation, écrire dans une autre langue. Caen: Paradigme. 